\title{
318 ENFORCED TUMOR SPECIFIC MHC-I HETEROGENEITY IN TRIPLE NEGATIVE BREAST CANCER DRIVES IMMUNOTHERAPY RESISTANCE
}

${ }^{1}$ Brandie Taylor*, ${ }^{2}$ Justin Balko, ${ }^{2}$ Melinda Sanders, ${ }^{2}$ Paula Gonzalez-Ericsson, ${ }^{2}$ Violeta Sanchez. 'Vanderbilt University, Nashville, TN, USA; ${ }^{2}$ Vanderbilt University Medical Center, Nashville, TN, USA

Background Despite the broad success of immune checkpoint inhibition (ICI e.g. anti-Programmed Death Ligand-1 [PD-L1]) in cancer treatment, tumor-intrinsic factors leading to intrinsic and acquired resistance are poorly understood. Tumor specific MHC-I expression is indispensable for anti-PD-1/L1 response as complete loss of MHC-I via B2M deletion results in inability of $\mathrm{CD} 8+\mathrm{T}$ cells to recognize tumor-associated antigens. However, MHC-I is heterogeneously downregulated or lost in many tumor types. Tumor cell destruction can also occur through non-synaptic mechanisms, in a so-called 'field effect'. Therefore, we modeled heterogeneous loss of MHC-I expression in breast cancer and experimentally evaluated how heterogeneous MHC-I loss affects response to anti-PD-L1 therapy.

Methods We performed quantitative immunofluorescence for MHC-I and Pan-CK on breast cancer tumors $(n=410)$. To determine the functional effect of MHC-I heterogeneity on anti-PD-L1 response, we used an immunocompetent EMT6 orthotopic mammary tumor model which ubiquitously expresses MHC-I at baseline. Using CRISPR/Cas9, we engineered EMT6 cells with B2m loci excision resulting in complete knockout of MHC-I on the cell surface. We then orthotopically implanted B2m-comtetent and B2m-KO cells at varying inoculum ratios (100:0, 90:10, 50:50, 10:90, 0:100) into syngeneic $\mathrm{Balb} / \mathrm{C}$ mice and assessed immune responsiveness and efficacy of checkpoint inhibition. Additionally, to look at how loss of MHC-I affects the tumor microenvironment we will use the PanCancer Immune NanoString panel $(n=770$ genes) to evaluate gene expression patterns in tumor cells and infiltrating immune cells.

Results In patient samples, we identified high diversity in MHC-I expression across all clinical subtypes, with triple negative breast cancer (TNBC) having the highest MHC-I expression. Chemotherapy-treated tumors had higher MHC-I levels than untreated tumors. In mice when $10 \%$ of cells were $\mathrm{B} 2 \mathrm{~m}$ $\mathrm{KO}$, we observed a $50 \%$ reduction in complete eradication of EMT6 tumors with aPD-L1 treatment and reduced disease-stabilization and no complete responses when a 50\% mixture of MHC-I deficient cells. An increasing percentage of $\mathrm{B} 2 \mathrm{~m} \mathrm{KO}$ leads to worse outcomes overall and a decrease in infiltrating T cells.

Conclusions Our work suggests that there is an ICI-responsive phenotype that is driven by heterogeneity in MHC-I expression levels. As little as $10 \%$ of tumor specific MHC-I loss can lead to therapeutic resistance and a decrease in complete responders. This represents that early TNBC may be less responsive to single-agent PD-L1 due to specific percentages of MHC-I loss. MHC-I expression can influence therapy outcomes and potentially lead to novel observations of how to overcome lack of, or limited, MHC-I expression.

http://dx.doi.org/10.1136/jitc-2021-SITC2021.318 